\title{
A Mean Field Type Flow on a Closed Riemannian Surface with the Action of an Isometric Group
}

\author{
Yamin Wang
}

\begin{abstract}
Let $(\Sigma, g)$ be a closed Riemannian surface, $\mathbf{G}=\left\{\sigma_{1}, \ldots, \sigma_{N}\right\}$ be an isometric group acting on it. Denote a positive integer $\ell=\min _{x \in \Sigma} I(x)$, where $I(x)$ is the number of all distinct points of the set $\left\{\sigma_{1}(x), \ldots, \sigma_{N}(x)\right\}$. By a method of flow due to Castéras (Pacific J. Math. 2015), we prove that the solution to the mean field equation

$$
-\Delta_{g} u=8 \pi \ell\left(\frac{h e^{u}}{\int_{\Sigma} h e^{u} d v_{g}}-\frac{1}{\operatorname{Vol}_{g}(\Sigma)}\right)
$$

exists under given conditions. This gives a new proof of Yang and Zhu's result in (Internat. J. Math. 2020). The case $\ell=1$ was studied by Li and Zhu (Calc. Var. Partial Differential Equations 2019).
\end{abstract}

\section{Introduction}

Let $(\Sigma, g)$ be a closed Riemannian surface and $\Delta$ be the Laplace-Beltrami operator with respect to the metric $g$. The famous mean field equation is stated as follows:

$$
-\Delta u=\rho\left(\frac{h e^{u}}{\int_{\Sigma} h e^{u} d v_{g}}-\frac{1}{\operatorname{Vol}_{g}(\Sigma)}\right),
$$

where $\rho$ is some real number, $h \in C^{\infty}(\Sigma)$, and $\operatorname{Vol}_{g}(\Sigma)$ stands for the volume of $\Sigma$. For $\rho<8 \pi$, Ding, Jost, Li and Wang [12 proved that (1.1) has a solution when $h$ is a smooth positive function; for $\rho=8 \pi$, a sufficient condition for existence of solutions to (1.1) is given by Yang and Zhu 23 when $h \geq 0$ and $h \not \equiv 0$. When $\Sigma$ is a flat torus, it was independently proved by Nolasco and Tarantello 20 that 1.1 has a solution for $\rho=8 \pi$. While the problem on $\mathbb{S}^{2}$ is much more complicated and known as the Nirenberg problem. For works in this direction, we refer the reader to $4,5,9,11,15,18,19]$. When $\rho \in\left(8 \pi, 4 \pi^{2}\right)$ and $h \equiv 1$, Struwe and Tarantello 22 pointed out that the solutions of 1.1 are nontrivial under the assumption that $\Sigma$ is flat torus with a fundamental domain. For $\rho \in(8 \pi, 16 \pi)$, it was proved by Ding, Jost, Li and Wang 13 that 1.1] exists a non-minimal solution. In the case $\rho \neq 8 N \pi, \forall N \in \mathbb{N}$, Chen and Lin 6, 7] obtained a degree-counting formula

Received August 5, 2020; Accepted January 17, 2021.

Communicated by Jenn-Nan Wang.

2020 Mathematics Subject Classification. 58J05.

Key words and phrases. mean field type flow, isometric group action, compactness. 
for (1.1) provided that the genus of $\Sigma$ is positive. Later, the result was generalized by Malchiodi 17 to $\rho \in(8 m \pi, 16 m \pi)\left(m \in \mathbb{Z}^{+}\right)$when $\Sigma$ is a general Riemannian surface. For the recent work, Li and Zhu 16 showed that under certain assumptions, (1.1) has a smooth solution with $\rho=8 \pi$ on a closed Riemannian surface.

Let $\mathbf{G}=\left\{\sigma_{1}, \ldots, \sigma_{N}\right\}$ be a finite isometric group acting on a closed Riemannian surface $(\Sigma, g)$, and $u: \Sigma \rightarrow \mathbb{R}$ be a measurable function, we say that $u \in \mathscr{I}_{\mathbf{G}}$ if $u$ is G-invariant, namely $u\left(\sigma_{i}(x)\right)=u(x)$ for any $1 \leq i \leq N$ and almost every $x \in \Sigma$. Define a Hilbert space

$$
\mathscr{H}_{\mathbf{G}}=\left\{u \in W^{1,2}(\Sigma, g) \cap \mathscr{I}_{\mathbf{G}}: \int_{\Sigma} u d v_{g}=0\right\}
$$

with an inner product $\langle u, v\rangle_{\mathscr{H}_{\mathrm{G}}}=\int_{\Sigma}\langle\nabla u, \nabla v\rangle d v_{g}$, where $\langle\nabla u, \nabla v\rangle$ stands for the Riemannian inner product of $\nabla u$ and $\nabla v$. Denote

$$
\ell=\min _{x \in \Sigma} I(x)
$$

with $I(x)=\sharp \mathbf{G}(x)$, where $\sharp A$ stands for the number of all distinct points in the set $A$, and $\mathbf{G}(x)=\left\{\sigma_{1}(x), \ldots, \sigma_{N}(x)\right\}$ for any $x \in \Sigma$. Recently, Yang and Zhu 25] extended Ding, Jost, Li and Wang's result 12 to $(\Sigma, g)$ with an isometric group action G. Precisely, for $\rho=8 \pi \ell$ and $u \in \mathscr{H}_{\mathbf{G}}$, they considered the functionals

$$
\widetilde{J}_{8 \pi \ell(1-\epsilon)}(u)=\frac{1}{2} \int_{\Sigma}\left|\nabla_{g} u\right|^{2} d v_{g}-8 \pi \ell(1-\epsilon) \log \int_{\Sigma} h e^{u} d v_{g},
$$

where $h$ is a smooth positive function and $h(\sigma(x))=h(x)$ for all $\sigma \in \mathbf{G}$ and all $x \in \Sigma$. For any $0<\epsilon<1$, it follows from Chen [8] and a direct method of variation that $\widetilde{J}_{8 \pi \ell(1-\epsilon)}$ attains its minimum at some minimizer $u_{\epsilon}$. While if $\widetilde{J}_{8 \pi \ell}$ has no minimizer on $\mathscr{H}_{\mathbf{G}}$, using a method of blow-up analysis, they obtain

$$
\inf _{u \in \mathscr{H}_{\mathbf{G}}} \widetilde{J}_{8 \pi \ell}(u) \geq-4 \pi \ell \max _{x \in \Sigma}\left(2 \log (\pi \ell h(x))+\widetilde{A}_{x}\right)-8 \pi \ell,
$$

where $\widetilde{A}_{x}=\lim _{r \rightarrow 0}\left(\widetilde{G}_{x}(y)+4 \log r\right)$ is a constant, $r$ denotes the geodesic distance between $x$ and $y, \widetilde{G}_{x}$ is a Green function satisfying

$$
\Delta_{g} \widetilde{G}_{x}=\frac{8 \pi \ell}{\operatorname{Vol}_{g}(\Sigma)}-8 \pi \sum_{i=1}^{\ell} \delta_{\sigma_{i}(x)} \text { and } \int_{\Sigma} \widetilde{G}_{x} d v_{g}=0
$$

Clearly, the minimizer is a solution of (1.1). Moreover, for works of related issues, we refer the reader to Fang and Yang 14 and Yang and Zhu 24].

Castéras [2] investigated a gradient flow related to the mean field equation (1.1). Continuing [2], Castéras [3] obtained the global existence of the flow. The mean field type 
flow in 2,3$]$ is presented as follows:

$$
\left\{\begin{array}{l}
\frac{\partial}{\partial t} e^{v}=\Delta v-Q+\rho \frac{e^{v}}{\int_{\Sigma} e^{v} d v_{g}} \\
v(x, 0)=v_{0}(x)
\end{array}\right.
$$

where $v_{0} \in C^{2+\alpha}(\Sigma), \alpha \in(0,1)$ is the initial data and $Q \in C^{\infty}(\Sigma)$ is a given function such that $\int_{\Sigma} Q d v_{g}=\rho$. It is a gradient flow involving the functional

$$
J_{\rho}(v(t))=\frac{1}{2} \int_{\Sigma}|\nabla v(t)|^{2} d v_{g}+\int_{\Sigma} Q v(t) d v_{g}-\rho \log \int_{\Sigma} e^{v(t)} d v_{g}
$$

Suppose $h \in C^{\infty}(\Sigma)$ is a positive function, and $h$ satisfies

$$
\Delta \log h=Q-\rho .
$$

Using the flow due to [2, 3], Li and Zhu [16] gave a new proof to the results of [12]. Motivated by [16, 25], it is natural for us to consider the same question as in 25] by the method of flow. Our aim is to prove the convergence of the mean field type flow (1.5) on $(\Sigma, g)$ with an isometric group action. Different from Yang and Zhu [25], it is not required to assume $\int_{\Sigma} v d v_{g}=0$ in our paper. Here we define a Hilbert space

$$
\mathscr{H}_{\mathbf{G}}^{n}=\left\{v \in W^{n, 2}(\Sigma, g) \cap \mathscr{I}_{\mathbf{G}}\right\}, \quad n=1,2,
$$

where $\mathscr{I}_{\mathbf{G}}$ is defined as in 1.2 .

Then our main result reads

Theorem 1.1. Let $(\Sigma, g)$ be a closed Riemannian surface, $\mathbf{G}=\left\{\sigma_{1}, \ldots, \sigma_{\ell}\right\}$ be an isometric group acting on it. Define a function space $\mathscr{H}_{\mathbf{G}}^{1}$ as in 1.8 and a function $I(x)$ as in (1.3). Let $v(t) \in \mathscr{H}_{\mathbf{G}}^{1}$ be the solution of 1.5 , and $Q$ be a smooth function in (1.6), satisfying $Q(\sigma(x))=Q(x)$ for all $\sigma \in \mathbf{G}$ and all $x \in \Sigma$. Suppose that $I(x) \equiv \ell$ for all $x \in \Sigma$, and that $2 \log h(x)+\widetilde{A}_{x}$ achieves its maximum at some point $p \in \Sigma$, where $h(x)$ and $\widetilde{A}_{x}$ are defined in (1.7) and (1.4) respectively. If in addition

$$
Q(p)>2 K(p)
$$

where $K(p)$ denotes the Gaussian curvature of $(\Sigma, g)$ at $p$, then for $\rho=8 \pi \ell$, there exists an initial data $v_{0} \in C^{2+\alpha}(\Sigma)$ such that $v(t)$ converges in $H^{2}(\Sigma)$ to a solution $v_{\infty} \in C^{\infty}(\Sigma)$ of

$$
-\Delta v_{\infty}+Q=8 \pi \ell \frac{e^{v_{\infty}}}{\int_{M} e^{v_{\infty}} d v_{g}} .
$$


The proof of Theorem 1.1 is based on the works of 2, 3, 16 related with a gradient flow. Let us describe its outline. To prove the convergence of the flow in 1.5 with $\rho=8 \pi \ell$, we first study some properties of the flow and then we get the compactness theorem. It is shown that we have the following alternative: either $v\left(t_{k}\right)$ is compact or $v\left(t_{k}\right)$ blows up, where $v\left(t_{k}\right)$ is a subsequence of $v(t)$ as $t_{k} \rightarrow \infty$. Next, we suppose blow-up happens. By blow-up analysis, we derive

$$
\begin{aligned}
\lim _{t \rightarrow+\infty} J_{8 \pi \ell}(v(t)) \geq & -4 \pi \ell \max _{x \in \Sigma}\left(2 \log (\pi \ell h(x))+\widetilde{A}_{x}\right)-8 \pi \ell \\
& +8 \pi \ell \int_{\Sigma} \log h d v_{g}-\frac{1}{2} \int_{\Sigma}|\nabla \log h|^{2} d v_{g}
\end{aligned}
$$

where $h(x)$ and $\widetilde{A}_{x}$ are defined in (1.7) and (1.4) respectively. However, under the hypothesis 1.9 , we construct a sequence of initial data $v_{0, \varepsilon}$ such that

$$
\begin{aligned}
J_{8 \pi \ell}\left(v_{0, \varepsilon}\right)< & -4 \pi \ell \max _{x \in \Sigma}\left(2 \log (\pi \ell h(x))+\widetilde{A}_{x}\right)-8 \pi \ell \\
& +8 \pi \ell \int_{\Sigma} \log h d v_{g}-\frac{1}{2} \int_{\Sigma}|\nabla \log h|^{2} d v_{g},
\end{aligned}
$$

which makes a contradiction, since $J_{8 \pi \ell}(v(t))$ decreases in $t$. Thus, we exclude the blow-up phenomenon. According to the monotonicity of $J_{8 \pi \ell}(v(t))$, under some appropriate initial data $v_{0, \varepsilon}$, we finally prove the solution of 1.5 converges to a solution $v_{\infty} \in C^{\infty}(\Sigma)$ of (1.10). Since the equation 1.10 is equivalent to the mean field equation $(1.1)$, we conclude that 1.1 has a smooth solution for $\rho=8 \pi \ell$. This ends the proof of Theorem 1.1. For the special case $\mathbf{G}=\{\mathrm{Id}\}$, where $\mathrm{Id}: \Sigma \rightarrow \Sigma$ is the identity map, our results are reduced to that of Li and Zhu [16]. Though the method we employ is similar to [16], there are many technical difficulties to be smoothed. Furthermore, by the symmetric properties of $(\Sigma, g)$, we deal with the singular points in constructing Green functions to derive the lower bound of $J_{8 \pi \ell}(v(t))$.

According to Yang and Zhu [24], one can raise the same question for the functional

$$
J_{\alpha, \beta}(u)=\frac{1}{2} \int_{\Sigma}\left(\left|\nabla_{g} u\right|^{2}-\alpha u^{2}\right) d v_{g}-\beta \log \int_{\Sigma} h e^{u} d v_{g}
$$

on a function space $\mathscr{H}=\left\{u \in W^{1,2}(\Sigma, g): \int_{\Sigma} u d v_{g}=0\right\}$. It is also interesting to consider the existence of solutions to (1.1) through the method of flow.

Note that $\frac{\partial}{\partial t} \int_{\Sigma} e^{v(t)} d v_{g}=0$ by 1.5 . This leads to $\int_{\Sigma} e^{v(t)} d v_{g}=C$. Hereafter, we can assume without loss of generality that $\int_{\Sigma} e^{v(t)} d v_{g}=1$. The remaining part of this paper is to prove Theorem 1.1. Throughout this paper, we assume the volume of $\Sigma$ equals to 1 , and we write $v_{k}=v\left(t_{k}\right)$ for simplicity. Moreover, sequence and subsequence are not distinguished, and various constants are often denoted by the same $C$ from line to line. 


\section{Proof of Theorem 1.1}

In this section, we begin by studying some properties of the flow. Following the same arguments of [3. Theorem 0.1], we can obtain the global solution of the flow (1.5) on a closed Riemann surface with an isometric group action. As an obvious analogue of Proposition 2.1 in [16], we prove

Proposition 2.1. Let $v(t) \in \mathscr{H}_{\mathbf{G}}^{1}$ be the solution of 1.5 with $\rho=8 \pi \ell$. For all $t \geq 0$, there holds

$$
J_{8 \pi \ell}(v(t)) \geq-C
$$

where $C>0$ is a constant not depending on $t$ and $\mathscr{H}_{\mathbf{G}}^{1}$ is defined in (1.8.

Proof. Denote $\bar{v}=\int_{\Sigma} v d v_{g}$. Since $\int_{\Sigma} Q d v_{g}=8 \pi \ell$, we have

$$
J_{8 \pi \ell}(v(t))=\frac{1}{2} \int_{\Sigma}|\nabla v(t)|^{2} d v_{g}+\int_{\Sigma} Q(v(t)-\bar{v}) d v_{g}-8 \pi \ell \log \int_{\Sigma} e^{v(t)-\bar{v}} d v_{g} .
$$

According to Chen [8, one gets by Young's inequality

$$
\log \int_{\Sigma} e^{v-\bar{v}} d v_{g} \leq \log \int_{\Sigma} e^{\frac{1}{16 \pi \ell}\|\nabla v\|_{2}^{2}+4 \pi \ell \frac{v^{2}}{\|\nabla v\|_{2}^{2}}} d v_{g} \leq \frac{1}{16 \pi \ell} \int_{\Sigma}|\nabla v|^{2} d v_{g}+C .
$$

Inserting (2.3) into 2.2 , we obtain

$$
J_{8 \pi \ell}(v(t)) \geq \int_{\Sigma} Q(v(t)-\bar{v}) d v_{g}-C .
$$

In view of (1.5) and (1.7), applying the integration by parts, one has

$$
\begin{aligned}
\int_{\Sigma} Q(v(t)-\bar{v}) d v_{g} & =\int_{\Sigma} \Delta v \cdot \log h d v_{g} \\
& =\int_{\Sigma} \frac{\partial e^{v}}{\partial t} \log h d v_{g}+\int_{\Sigma}\left(Q-8 \pi \ell e^{v}\right) \log h d v_{g}
\end{aligned}
$$

We estimate the two integrals on the right-hand side of 2.5 respectively. Taking the derivative with respect to $t$ of $J_{8 \pi \ell}(v(t))$ in $(1.6)$, one can check that

$$
\frac{\partial}{\partial t} J_{8 \pi \ell}(v(t))=\int_{\Sigma}\left(-\Delta v+Q-8 \pi \ell e^{v}\right) \frac{\partial v}{\partial t} d v_{g}=-\int_{\Sigma}\left(\frac{\partial v}{\partial t}\right)^{2} e^{v} d v_{g}
$$

due to 1.5 . Since $Q \in C^{\infty}(\Sigma)$ and $h \in C^{\infty}(\Sigma)$, it follows from the Hölder inequality and (2.6) that

$$
\begin{aligned}
\int_{\Sigma} \frac{\partial e^{v}}{\partial t} \log h d v_{g} & \geq-\max _{\Sigma}|\log h|\left(\int_{\Sigma}\left(\frac{\partial v}{\partial t}\right)^{2} e^{v} d v_{g}\right)^{1 / 2} \\
& =-\max _{\Sigma}|\log h|\left(-\frac{\partial}{\partial t} J_{8 \pi \ell}(v(t))\right)^{1 / 2}
\end{aligned}
$$


and that

$$
\int_{\Sigma}\left(Q-8 \pi \ell e^{v}\right) \log h d v_{g} \geq \int_{\Sigma} Q \log h d v_{g}-8 \pi \ell \max _{\Sigma}|\log h| .
$$

Combing (2.4), 2.5), 2.7) and (2.8), we obtain

$$
J_{8 \pi \ell}(v(t)) \geq-8 \pi \ell \max _{\Sigma}|\log h|+\int_{\Sigma} Q \log h d v_{g}-\max _{\Sigma}|\log h|\left(-\frac{\partial}{\partial t} J_{8 \pi \ell}(v(t))\right)^{1 / 2}-C
$$

If $\max _{\Sigma}|\log h|=0$, we can get the desired result directly. In the following, suppose $\max _{\Sigma}|\log h|>0$. Then $(2.9)$ can be rewritten as

$$
\frac{J_{8 \pi \ell}(v(t))}{\max _{\Sigma}|\log h|} \geq-8 \pi \ell+\frac{\int_{\Sigma} Q \log h d v_{g}-C}{\max _{\Sigma}|\log h|}-\left(-\frac{\partial}{\partial t} J_{8 \pi \ell}(v(t))\right)^{1 / 2} .
$$

Denote

$$
\xi=\frac{1}{\max _{\Sigma}|\log h|}, \quad \zeta=-8 \pi \ell+\frac{\int_{\Sigma} Q \log h d v_{g}-C}{\max _{\Sigma}|\log h|} .
$$

We claim that for any $t \geq 0$, there holds

$$
\xi J_{8 \pi \ell}(v(t))-\zeta \geq 0
$$

For otherwise, there exists some $t_{0}>0$ such that for all $t \geq t_{0}$,

$$
\xi J_{8 \pi \ell}(v(t))-\zeta<0
$$

By $(2.10)$, we have for any $t_{1} \geq t_{0}$ that

$$
\int_{t_{0}}^{t_{1}}-\frac{d J_{8 \pi \ell}(v(t))}{\left(-\xi J_{8 \pi \ell}(v(t))+\zeta\right)^{2}} \geq t_{1}-t_{0}
$$

namely,

$$
\left(-\xi\left(t_{1}-t_{0}\right)+\frac{1}{-\xi J_{8 \pi \ell}\left(v\left(t_{0}\right)\right)+\zeta}\right)\left(-\xi J_{8 \pi \ell}\left(v\left(t_{1}\right)\right)+\zeta\right) \geq 1 .
$$

Letting $t_{1} \rightarrow+\infty$, we find

$$
\lim _{t \rightarrow+\infty} J_{8 \pi \ell}(v(t)) \geq \frac{\xi}{\zeta}
$$

which contradicts 2.13 . Then, inserting 2.11 into 2.12), one gets by 1.7

$$
J_{8 \pi \ell}(v(t)) \geq 8 \pi \ell \int_{\Sigma} \log h d v_{g}-\int_{\Sigma}|\nabla \log h|^{2} d v_{g}-8 \pi \ell \max _{\Sigma}|\log h|-C .
$$

Noting that $h \in C^{\infty}(\Sigma)$, we conclude (2.1). This completes the proof. 
In view of (2.6), we can see that $J_{8 \pi \ell}(v(t))$ decreases with respect to $t$. By integrating (2.6) from 0 to $t$, one finds

$$
\int_{0}^{t} \int_{\Sigma}\left(\frac{\partial v}{\partial t}\right)^{2} e^{v} d v_{g} d t=J_{8 \pi \ell}(v(0))-J_{8 \pi \ell}(v(t)) .
$$

This together with Proposition 2.1 leads to

$$
\int_{0}^{\infty} \int_{\Sigma}\left(\frac{\partial v}{\partial t}\right)^{2} e^{v} d v_{g} d t<C<+\infty
$$

Thus, there exists a sequence $t_{k} \rightarrow+\infty$ such that

$$
\lim _{t_{k} \rightarrow+\infty} \int_{\Sigma}\left(\frac{\partial v_{k}}{\partial t}\right)^{2} e^{v_{k}} d v_{g} \rightarrow 0
$$

as $k \rightarrow+\infty$.

To proceed, we need the following estimate, which is similar to Proposition 2.1 in 3 . Proposition 2.2. For $\rho=8 \pi \ell$, if $v(t) \in \mathscr{H}_{\mathbf{G}}^{1}$ is the solution of $(1.5)$, then

$$
-\frac{\partial}{\partial t} e^{v(x, t)}+8 \pi \ell e^{v(x, t)} \geq-C, \quad \forall t \geq 0, \forall x \in \Sigma,
$$

where the constant $C>0$ not depending on $t$, and $\mathscr{H}_{\mathbf{G}}^{1}$ is defined in 1.8 .

Since no new idea comes out in its proof, we omit the details here but refer the readers to [3. Thanks to 2.14 and 2.15), we can see that the conditions in [2, Theorem 1.2] are satisfied by $v_{k}$. Following [2], we describe the compactness theorem as below.

Theorem 2.3. Define a function space $\mathscr{H}_{\mathbf{G}}^{1}$ as in $(1.8)$. Let $v(t) \in \mathscr{H}_{\mathbf{G}}^{1}$ be the solution of (1.5) with $\rho=8 \pi \ell$. Then for a sequence $t_{k} \rightarrow+\infty$, we have for $k \rightarrow+\infty$, either

(i) there exists a constant $C$ not depending on $k$ such that

$$
\left\|v_{k}\right\|_{H^{2}(\Sigma)} \leq C
$$

or (ii) there exists a sequence of points $\left\{x_{k}\right\}$ and a sequence of real positive numbers $\left\{R_{k}\right\} \rightarrow 0$ such that

$$
\lim _{k \rightarrow+\infty} \int_{B_{2 R_{k}\left(\sigma_{i}\left(x_{k}\right)\right)}} e^{v_{k}} d v_{g}=\frac{1}{\ell}, \quad \forall i=1, \ldots, \ell,
$$

where $B_{2 R_{k}\left(\sigma_{i}\left(x_{k}\right)\right)} \subset \Sigma$ denotes a geodesic ball centered at $\sigma_{i}\left(x_{k}\right)$ with radius $2 R_{k}$, and

$$
\lim _{k \rightarrow+\infty} \int_{\Sigma \backslash \bigcup_{i=1}^{\ell} B_{2 R_{k}\left(\sigma_{i}\left(x_{k}\right)\right)}} e^{v_{k}} d v_{g}=0
$$

In what follows, the sequence $v_{k} \subseteq H^{2}(\Sigma)$ is said to be compact if it is uniformly bounded in $H^{2}(\Sigma)$. Theorem 2.3 shows that we have the following alternative: either $v_{k}$ is compact or $v_{k}$ blows up. Subsequently, we will exclude the blow-up phenomenon to occur. 


\section{Blow-up analysis}

Recall that $\mathscr{H}_{\mathbf{G}}^{2}$ is defined in $(1.8)$. In this section, we study the asymptotic behavior of non-compact solutions $v_{k} \subseteq \mathscr{H}_{\mathbf{G}}^{2}$ in Theorem 2.3. Set

$$
\Delta v_{k}=Q+\frac{\partial e^{v_{k}}}{\partial t}-8 \pi \ell e^{v_{k}}:=F_{k}
$$

where $\int_{\Sigma} e^{v_{k}} d v_{g}=1$. Similar to [16, we discuss the convergence of $v_{k}$ near and away from the blow-up point $x_{0}$. Based on the blow-up analysis, we finally calculate

$$
\begin{aligned}
\lim _{t \rightarrow+\infty} J_{8 \pi \ell}(v(t)) \geq & -4 \pi \ell \max _{x \in \Sigma}\left(2 \log (\pi \ell h(x))+\widetilde{A}_{x}\right)-8 \pi \ell \\
& +8 \pi \ell \int_{\Sigma} \log h d v_{g}-\frac{1}{2} \int_{\Sigma}|\nabla \log h|^{2} d v_{g}
\end{aligned}
$$

where $h(x)$ and $\widetilde{A}_{x}$ are defined in $(1.7)$ and $(1.4)$ respectively.

\subsection{Asymptotic behavior near the blow-up point}

According to [2, Proposition 3.1], the convergence of $v_{k}$ is described as follows:

Proposition 3.1. Let $v_{k} \subseteq \mathscr{H}_{\mathbf{G}}^{2}$ be a sequence of non-compact solutions of (3.1), satisfying (2.14) and 2.15). Denote

$$
\widetilde{v}_{k}=v_{k}\left(\exp _{x_{k}}\left(r_{k} \cdot\right)\right)+2 \log r_{k}
$$

where $\exp _{x_{k}}$ represents the exponential map centered in $x_{k}$ and $\mathscr{H}_{\mathbf{G}}^{2}$ is in $(1.8)$. Then, there exist a sequence of points $x_{k}$ and a sequence of real numbers $r_{k}$ such that as $k \rightarrow+\infty$, $\widetilde{v}_{k} \rightarrow \widetilde{v}_{\infty}$ in $C_{\text {loc }}^{\alpha}\left(\mathbb{R}^{2}\right)$ for some $\alpha \in(0,1)$, and weakly in $H_{\text {loc }}^{2}\left(\mathbb{R}^{2}\right)$, where $\widetilde{v}_{\infty}$ is the solution of

$$
-\Delta \widetilde{v}_{\infty}=8 \pi \ell e^{\widetilde{v}_{\infty}}
$$

Moreover, there exist $\lambda>0$ and $\widetilde{x}_{0} \in \mathbb{R}^{2}$ such that

$$
\widetilde{v}_{\infty}(x)=2 \log \frac{2 \lambda}{1+\left(\lambda\left|x-\widetilde{x}_{0}\right|\right)^{2}}+\log \frac{1}{4 \pi \ell} .
$$

Since the proof of Proposition 3.1 is an obvious analog of that of [2, Proposition 3.1], we omit it, but refer the reader to 2 for details.

\subsection{Convergence away from the blow-up point}

Similar to [16], we have the following two observations of $v_{k}$, which are essential in our analysis. The difference is that $v_{k}$ is $\mathbf{G}$-invariant in our case, namely $v_{k}\left(\sigma_{i}(x)\right)=v_{k}(x)$ for any $1 \leq i \leq N$ and almost every $x \in \Sigma$. The first key observation is the following: 
Proposition 3.2. Let $v_{k} \subseteq \mathscr{H}_{\mathbf{G}}^{2}$ be a sequence of non-compact solutions of (3.1), satisfying (2.14) and 2.15). Then for any $1<p<2$, there holds

$$
\left\|v_{k}-\bar{v}_{k}\right\|_{W^{1, p}(\Sigma)} \leq C
$$

where the constant $C>0$ is independent of $k$, and $\mathscr{H}_{\mathbf{G}}^{2}$ is defined in 1.8 .

Proof. By the result of [25, Proposition 5], there exists a unique Green function $\widetilde{G}_{x}(y)$ on $(\Sigma, g)$, which is a distributional solution to

$$
\Delta_{g} \widetilde{G}_{x}=\sum_{i=1}^{\ell} \delta_{\sigma_{i}(x)}-\ell
$$

Note that $v_{k}(\sigma(x))=v_{k}(x)$ for all $\sigma \in \mathbf{G}$ and all $x \in \Sigma$. Then it follows from [1, Theorem 4.13] that

$$
v_{k}(x)-\bar{v}_{k}=\frac{1}{\ell} \int_{\Sigma} \widetilde{G}_{x}(y) F_{k}(y) d v_{g}(y) \quad \text { for a.e. } x \in \Sigma,
$$

and that

$$
\left|\nabla v_{k}(x)\right| \leq \frac{1}{\ell} \int_{\Sigma}\left|\nabla \widetilde{G}_{x}(y)\right|\left|F_{k}(y)\right| d v_{g}(y) \leq C \int_{\Sigma} \frac{1}{|x-y|}\left|F_{k}(y)\right| d v_{g}(y) .
$$

Combing 2.15) and (3.1), we deduce that

$$
\left\|F_{k}\right\|_{L^{1}(\Sigma)} \leq 16 \pi \ell+\left\|\frac{\partial e^{v_{k}}}{\partial t}\right\|_{L^{1}(\Sigma)} \leq C .
$$

This together with (3.4), Jensen's inequality and Fubini's Theorem gives

$$
\begin{aligned}
\int_{B_{r}\left(x^{*}\right)}\left|\nabla v_{k}(x)\right|^{p} d v_{g}(x) & \leq \int_{B_{r}\left(x^{*}\right)} \int_{\Sigma}\left\|F_{k}\right\|_{L^{1}(\Sigma)}^{p-1} \frac{\left|F_{k}(y)\right|}{|x-y|^{p}} d v_{g}(y) d v_{g}(x) \\
& \leq C \sup _{y \in \Sigma} \int_{B_{r}\left(x^{*}\right)} \frac{1}{|x-y|^{p}} d v_{g}(x) \\
& \leq C r^{2-p}
\end{aligned}
$$

where $B_{r}\left(x^{*}\right) \subset \Sigma$ denotes a ball centered at $x^{*}$ with radius $r>0$. Noticing $\Sigma$ is compact, for any $1<p<2$, we can see $\left\|\nabla v_{k}(x)\right\|_{L^{p}(\Sigma)} \leq C$ by $(3.6)$. Then by Poincaré's inequality, we get the desired result. This achieves the proof of the proposition.

To get the convergence of $v_{k}$ away from the blow-up point, we also need the proposition as below.

Proposition 3.3. Let $v_{k} \subseteq \mathscr{H}_{\mathbf{G}}^{2}$ be a sequence of non-compact solutions of (3.1), satisfying (2.14) and 2.15). Then for each $V \subset \subset \Sigma \backslash\left\{\bigcup_{i=1}^{\ell} \sigma_{i}\left(x_{0}\right)\right\}$, there exist constants $C>0$ and $\alpha>1$ such that

$$
\int_{V} e^{\alpha\left(v_{k}-\bar{v}_{k}\right)} d v_{g} \leq C
$$

where $\mathscr{H}_{\mathbf{G}}^{2}$ is defined in 1.8 . 
Proof. Let $V$ be any subset satisfying $V \subset \subset \Sigma \backslash\left\{\bigcup_{i=1}^{\ell} \sigma_{i}\left(x_{0}\right)\right\}$. Note that $v_{k}$ is noncompact. By the results of [2, Proposition 2.1] and Theorem 2.3, we have for any $x \in V$, there exists $r_{x}>0$ such that for some $\delta_{x}>0$

$$
\int_{B_{r_{x}}(x)}\left|F_{k}\right|<4 \pi-\delta_{x}
$$

in $B_{r_{x}}(x) \subset \Sigma \backslash\left\{\bigcup_{i=1}^{\ell} \sigma_{i}\left(x_{0}\right)\right\}$. Then we can find an integer $m$ satisfying $\bar{V} \subset \bigcup_{j=1}^{m} B_{r_{x_{j}} / 2}\left(x_{j}\right)$, where $x_{j} \in V$. In view of 3.3 , for $x \in B_{r_{x_{j}} / 2}\left(x_{j}\right)$, one has by (3.5) that

$$
\begin{aligned}
e^{\alpha\left(v_{k}(x)-\bar{v}_{k}\right)} & =e^{\frac{\alpha}{\ell}\left(\int_{B_{r_{j}}\left(x_{j}\right)} \widetilde{G}_{x}(y) F_{k}(y) d v_{g}(y)+\int_{\Sigma \backslash B_{r_{x_{j}}}\left(x_{j}\right)} \widetilde{G}_{x}(y) F_{k}(y) d v_{g}(y)\right)} \\
& \leq C e^{\frac{\alpha}{\ell} \int_{B_{r_{x_{j}}}\left(x_{j}\right)} \widetilde{G}_{x}(y) F_{k}(y) d v_{g}(y)},
\end{aligned}
$$

where $\alpha>0$ is a constant and $\widetilde{G}_{x}(y)$ is in 3.2 .

Set $\beta(y)=\left|F_{k}(y) \chi_{B_{r_{x_{j}}}\left(x_{j}\right)}\right| /\left\|F_{k}(y) \chi_{B_{r_{x_{j}}}\left(x_{j}\right)}\right\|_{L^{1}(\Sigma)}$. This together with 3.8 yields

$$
\begin{aligned}
& \int_{B_{r_{x_{j}} / 2}\left(x_{j}\right)} e^{\alpha\left(v_{k}(x)-\bar{v}_{k}\right)} d v_{g}(x) \\
& \leq C \int_{B_{r_{x_{j}} / 2}\left(x_{j}\right)} \int_{\Sigma} \beta(y) e^{\frac{\alpha}{\ell}\left\|F_{k}(y) \chi_{B_{r_{x_{j}}}\left(x_{j}\right)}\right\|_{L^{1}(\Sigma)}\left|\widetilde{G}_{x}(y)\right|} d v_{g}(y) d v_{g}(x) \\
& \leq C \sup _{y \in \Sigma} \int_{\Sigma}\left(\frac{1}{|x-y|}\right)^{\frac{\alpha}{2 \pi \ell}\left\|F_{k}(y) \chi_{B_{r_{j}}}\left(x_{j}\right)\right\|_{L^{1}(\Sigma)}} d v_{g}(x) \text {. }
\end{aligned}
$$

The first inequality is a direct consequence of Jensen's inequality. The second one follows from [1, Theorem 4.13]. Due to (3.7) and $\ell \geq 1$, there exists the constant $\alpha>1$ such that

$$
\frac{\alpha}{2 \pi \ell}\left\|F_{k}(y) \chi_{B_{r_{x_{j}}}\left(x_{j}\right)}\right\|_{L^{1}(\Sigma)}<2
$$

for each $j \in\{1, \ldots, m\}$. As a consequence,

$$
\int_{V} e^{\alpha\left(v_{k}-\bar{v}_{k}\right)} d v_{g} \leq \sum_{j=1}^{m} \int_{B_{r_{x_{j}} / 2}\left(x_{j}\right)} e^{\alpha\left(v_{k}(x)-\bar{v}_{k}\right)} d v_{g}(x) \leq C .
$$

Therefore, Proposition 3.3 is established.

Recall that $h$ is defined as in (1.7). Denote $\mu_{k}=v_{k}-\log h$. It is clear that

$$
\Delta\left(\mu_{k}-\bar{\mu}_{k}\right)=8 \pi \ell+\frac{\partial e^{v_{k}}}{\partial t}-8 \pi \ell e^{v_{k}},
$$

and that $\mu_{k} \subseteq \mathscr{H}_{\mathbf{G}}^{2}$. Then we obtain the proposition as follows. 
Proposition 3.4. Let $\mu_{k}$ be defined as above. For $1<p<2$ and some $0<\gamma<1$, there holds

$$
\begin{cases}\mu_{k}-\bar{\mu}_{k} \rightarrow \widetilde{G}_{x_{0}} & \text { weakly in } W^{1, p}(\Sigma) \\ \mu_{k}-\bar{\mu}_{k} \rightarrow \widetilde{G}_{x_{0}} & \text { strongly in } W_{\mathrm{loc}}^{2,2}\left(\Sigma \backslash\left\{\bigcup_{i=1}^{\ell} \sigma_{i}\left(x_{0}\right)\right\}\right), \\ \mu_{k}-\bar{\mu}_{k} \rightarrow \widetilde{G}_{x_{0}} & \text { in } C_{\mathrm{loc}}^{\gamma}\left(\Sigma \backslash\left\{\bigcup_{i=1}^{\ell} \sigma_{i}\left(x_{0}\right)\right\}\right)\end{cases}
$$

as $k \rightarrow+\infty$, where the Green function $\widetilde{G}_{x_{0}}$ satisfies

$$
\Delta \widetilde{G}_{x_{0}}=8 \pi \ell-8 \pi \sum_{i=1}^{\ell} \delta_{\sigma_{i}\left(x_{0}\right)} \quad \text { and } \quad \int_{\Sigma} \widetilde{G}_{x_{0}} d v_{g}=0
$$

Moreover, $\widetilde{G}_{x_{0}}$ takes the form

$$
\widetilde{G}_{x_{0}}(x)=-4 \log r+\widetilde{A}_{x_{0}}+O(r)
$$

near $\sigma_{i}\left(x_{0}\right)$, where $\widetilde{A}_{x_{0}}$ is a constant, $r$ denotes the geodesic distance between $x$ and $\sigma_{i}\left(x_{0}\right)$, $i=1, \ldots, \ell$.

Proof. Observe that $\log h \in C^{\infty}(\Sigma)$. By employing Proposition 3.2, we see that $\| \mu_{k}-$ $\bar{\mu}_{k} \|_{W^{1, p}(\Sigma)} \leq C$ for any $1<p<2$. Since $\widetilde{G}_{x_{0}}(x)$ is the unique solution of (3.10), up to a subsequence, we have $\mu_{k}-\bar{\mu}_{k} \rightarrow \widetilde{G}_{x_{0}}$ weakly in $W^{1, p}(\Sigma)$ as $k \rightarrow+\infty$. Recall that $V \subset \subset \Sigma \backslash\left\{\bigcup_{i=1}^{\ell} \sigma_{i}\left(x_{0}\right)\right\}$. Due to Proposition 3.3. we obtain by using the Jensen's inequality that

$$
\int_{V} e^{\alpha v_{k}(x)} d v_{g}=e^{\alpha \bar{v}_{k}} \int_{V} e^{\alpha\left(v_{k}(x)-\bar{v}_{k}\right)} d v_{g} \leq C\left(\int_{\Sigma} e^{v_{k}(x)} d v_{g}\right)^{\alpha} \leq C,
$$

where $\alpha>1$. Together with Hölder's inequality and (2.14), it leads to

$$
\int_{V}\left|\frac{\partial e^{v_{k}}}{\partial t}\right|^{r} d v_{g} \leq\left(\int_{V}\left(\frac{\partial v_{k}}{\partial t}\right)^{2} e^{v_{k}} d v_{g}\right)^{r / 2}\left(\int_{V} e^{\alpha v_{k}(x)} d v_{g}\right)^{1-r / 2} \rightarrow 0
$$

as $k \rightarrow+\infty$, where $r=2 \alpha /(\alpha+1)>1$. Choose $\omega=\min \{r, \alpha\}$. Combining (3.12) and (3.13), we employ the elliptic estimate to (3.9), which yields $\left\|\mu_{k}-\bar{\mu}_{k}\right\|_{W_{\text {loc }}^{2, \omega}(V)} \leq C$. And then Sobolev's embedding theorem implies that $\mu_{k}-\bar{\mu}_{k} \rightarrow \widetilde{G}_{x_{0}}$ in $C_{\text {loc }}^{\gamma}\left(\Sigma \backslash\left\{\bigcup_{i=1}^{\ell} \sigma_{i}\left(x_{0}\right)\right\}\right)$ for $0<\gamma<1$. Following the same arguments as in [16, Proposition 3.5], one can show that $\left\|\mu_{k}-\bar{\mu}_{k}-\widetilde{G}_{x_{0}}\right\|_{H^{2}(V)} \rightarrow 0$. By elliptic estimates, we obtain (3.11). This concludes the proof of Proposition 3.4 .

\subsection{A lower bound of $J_{8 \pi \ell}(v(t))$}

In this subsection, we shall derive a lower bound of $J_{8 \pi \ell}(v(t))$. Precisely, we have the following proposition. 
Proposition 3.5. Define a function space $\mathscr{H}_{\mathbf{G}}^{1}$ as in $(1.8)$. Let $v(t) \in \mathscr{H}_{\mathbf{G}}^{1}$ be the solution of (1.5) with $\rho=8 \pi \ell$. Suppose $v_{k}$ is a noncompact sequence of $v(t)$. Then we have

$$
\begin{aligned}
\lim _{t \rightarrow+\infty} J_{8 \pi \ell}(v(t)) \geq & -4 \pi \ell \max _{x \in \Sigma}\left(2 \log (\pi \ell h(x))+\widetilde{A}_{x}\right)-8 \pi \ell \\
& +8 \pi \ell \int_{\Sigma} \log h d v_{g}-\frac{1}{2} \int_{\Sigma}|\nabla \log h|^{2} d v_{g},
\end{aligned}
$$

where where $h(x)$ and $\widetilde{A}_{x}$ are defined in (1.7) and (3.11) respectively.

Proof. We prove the statement on the contrary. For otherwise, there exists a constant $\varepsilon>0$ such that

$$
\begin{aligned}
\lim _{t \rightarrow+\infty} J_{8 \pi \ell}(v(t))< & -4 \pi \ell \max _{x \in \Sigma}\left(2 \log (\pi \ell h(x))+\widetilde{A}_{x}\right)-8 \pi \ell \\
& +8 \pi \ell \int_{\Sigma} \log h d v_{g}-\frac{1}{2} \int_{\Sigma}|\nabla \log h|^{2} d v_{g}-\varepsilon .
\end{aligned}
$$

Let $v_{k} \subseteq \mathscr{H}_{\mathbf{G}}^{2}$ be a sequence of non-compact solutions of (3.1), satisfying (2.14) and (2.15). Notice that $\int_{\Sigma} e^{v_{k}}=1$. This leads to $\log \int_{\Sigma} e^{v_{k}} d v_{g}=0$. Then 1.6 can be rewritten as

$$
J_{8 \pi \ell}\left(v_{k}\right)=\frac{1}{2} \int_{\Sigma}\left|\nabla v_{k}\right|^{2} d v_{g}+\int_{\Sigma} Q v_{k} d v_{g}
$$

where $\int_{\Sigma} Q d v_{g}=8 \pi \ell$. Now, we estimate the two integrals on the right-hand side of (3.16) respectively. By Proposition 3.4.

$$
v_{k}-\bar{v}_{k} \rightarrow \widetilde{G}_{x_{0}}+\log h-\int_{\Sigma} \log h d v_{g}
$$

in $C_{\text {loc }}^{\gamma}\left(\Sigma \backslash\left\{\bigcup_{i=1}^{\ell} \sigma_{i}\left(x_{0}\right)\right\}\right)$, for some $0<\gamma<1$. This together with 1.7 gives that

$$
\begin{aligned}
\int_{\Sigma} Q v_{k} d v_{g} & =-\int_{\Sigma} \nabla \log h \cdot \nabla v_{k} d v_{g}+8 \pi \ell \bar{v}_{k} \\
& =-\int_{\Sigma}|\nabla \log h|^{2} d v_{g}-\int_{\Sigma} \nabla \log h \cdot \nabla \widetilde{G}_{x_{0}} d v_{g}+8 \pi \ell \bar{v}_{k}+o_{k}(1) .
\end{aligned}
$$

To calculate the integral $\int_{\Sigma}\left|\nabla v_{k}\right|^{2} d v_{g}$, we divide it into three parts, namely

$$
\begin{aligned}
& \int_{\Sigma \backslash \bigcup_{i=1}^{\ell} B_{\delta}\left(\sigma_{i}\left(x_{k}\right)\right)}\left|\nabla v_{k}\right|^{2} d v_{g}+\int_{\bigcup_{i=1}^{\ell} B_{\delta}\left(\sigma_{i}\left(x_{k}\right)\right) \backslash B_{R r_{k}}\left(\sigma_{i}\left(x_{k}\right)\right)}\left|\nabla v_{k}\right|^{2} d v_{g} \\
+ & \int_{\bigcup_{i=1}^{\ell} B_{R r_{k}}\left(\sigma_{i}\left(x_{k}\right)\right)}\left|\nabla v_{k}\right|^{2} d v_{g},
\end{aligned}
$$

where $\delta>0$. Then the above parts shall be estimated respectively. We begin with the first part. It follows from Proposition 3.4 that

$$
\begin{aligned}
& \int_{\Sigma \backslash \bigcup_{i=1}^{\ell} B_{\delta}\left(\sigma_{i}\left(x_{k}\right)\right)}\left|\nabla v_{k}\right|^{2} d v_{g} \\
= & \int_{\Sigma \backslash \bigcup_{i=1}^{\ell} B_{\delta}\left(\sigma_{i}\left(x_{k}\right)\right)}\left|\nabla\left(\mu_{k}+\log h\right)\right|^{2} d v_{g} \\
= & \int_{\Sigma \backslash \bigcup_{i=1}^{\ell} B_{\delta}\left(\sigma_{i}\left(x_{0}\right)\right)}\left(\left|\nabla \widetilde{G}_{x_{0}}\right|^{2}+2 \nabla \widetilde{G}_{x_{0}} \cdot \nabla \log h+|\nabla \log h|^{2}\right) d v_{g}+o_{k}(1) .
\end{aligned}
$$


In a normal coordinate system $\left\{x_{1}, x_{2}\right\}$ near $x_{0}$, by elliptic estimates, $\widetilde{G}_{x_{0}}$ can be represented by

$$
\widetilde{G}_{x_{0}}(x)=-4 \log r+\widetilde{A}_{x_{0}}+b_{1} x_{1}+b_{2} x_{2}+c_{1} x_{1}^{2}+2 c_{2} x_{1} x_{2}+c_{3} x_{2}^{2}+O\left(r^{3}\right),
$$

where $\widetilde{A}_{x_{0}}, b_{1}, b_{2}, c_{1}, c_{2}, c_{3}$ are constants, $r(x)$ denotes the geodesic distance between $x$ and $\sigma_{i}\left(x_{0}\right), i=1, \ldots, \ell$. Using the divergence theorem, we calculate by 3.10 and 3.20 that

$$
\begin{aligned}
\int_{\Sigma \backslash \bigcup_{i=1}^{\ell} B_{\delta}\left(\sigma_{i}\left(x_{0}\right)\right)}\left|\nabla \widetilde{G}_{x_{0}}\right|^{2} d v_{g}= & -\int_{\bigcup_{i=1}^{\ell} \partial B_{\delta}\left(\sigma_{i}\left(x_{0}\right)\right)} \widetilde{G}_{x_{0}} \cdot \frac{\partial \widetilde{G}_{x_{0}}}{\partial n} d s_{g} \\
& -\int_{\Sigma \backslash \bigcup_{i=1}^{\ell} B_{\delta}\left(\sigma_{i}\left(x_{0}\right)\right)} \widetilde{G}_{x_{0}}\left(8 \pi \ell-8 \pi \sum_{i=1}^{\ell} \delta_{\sigma_{i}\left(x_{0}\right)}\right) d v_{g} \\
= & -32 \pi \ell \log \delta+8 \pi \ell \widetilde{A}_{x_{0}}+o_{\delta}(1) .
\end{aligned}
$$

Inserting (3.21) into 3.19 , one has

$$
\begin{aligned}
& \int_{\Sigma \backslash \bigcup_{i=1}^{\ell} B_{\delta}\left(\sigma_{i}\left(x_{k}\right)\right)}\left|\nabla v_{k}\right|^{2} d v_{g} \\
= & \int_{\Sigma \backslash \bigcup_{i=1}^{\ell} B_{\delta}\left(\sigma_{i}\left(x_{0}\right)\right)}\left(2 \nabla \widetilde{G}_{x_{0}} \cdot \nabla \log h d v_{g}+|\nabla \log h|^{2}\right) d v_{g} \\
& -32 \pi \ell \log \delta+8 \pi \ell \widetilde{A}_{x_{0}}+o_{\delta}(1)+o_{k}(1) .
\end{aligned}
$$

Next we estimate the integral of $v_{k}$ on the annulus. Since $v_{k} \subseteq \mathscr{H}_{\mathbf{G}}^{2}$, it yields to

$$
\int_{\bigcup_{i=1}^{\ell} B_{\delta}\left(\sigma_{i}\left(x_{k}\right)\right) \backslash B_{R r_{k}}\left(\sigma_{i}\left(x_{k}\right)\right)}\left|\nabla v_{k}\right|^{2} d v_{g}=\ell \int_{B_{\delta}\left(x_{k}\right) \backslash B_{R r_{k}}\left(x_{k}\right)}\left|\nabla v_{k}\right|^{2} d v_{g} .
$$

We use a technique of [12] to get the estimate on the annulus. Set

$$
\phi_{k}=\inf _{\partial B_{R r_{k}}\left(x_{k}\right)} v_{k}, \quad \psi_{k}=\sup _{\partial B_{\delta}\left(x_{k}\right)} v_{k}, \quad \varphi_{k}=\phi_{k}-\psi_{k}+2 \log r_{k}+\bar{v}_{k} .
$$

In view of Proposition 3.1 and $(3.17$, we see that as $k \rightarrow+\infty$

$$
\phi_{k}+2 \log r_{k} \rightarrow \inf _{|x|=R} \widetilde{v}_{\infty}(x) \quad \text { in } C_{\mathrm{loc}}^{\alpha}\left(\mathbb{R}^{2}\right),
$$

and

$$
\psi_{k}-\bar{v}_{k} \rightarrow \sup _{\partial B_{\delta}\left(x_{0}\right)}\left(\widetilde{G}_{x_{0}}+\log h-\int_{\Sigma} v \log h d v_{g}\right) \quad \text { in } C_{\mathrm{loc}}^{\gamma}\left(\Sigma \backslash\left\{\bigcup_{i=1}^{\ell} \sigma_{i}\left(x_{0}\right)\right\}\right),
$$

where $0<\alpha<1$ and $0<\gamma<1$. Then,

$$
\varphi_{k} \rightarrow \inf _{|x|=R} \widetilde{v}_{\infty}(x)-\sup _{\partial B_{\delta}\left(x_{0}\right)}\left(\widetilde{G}_{x_{0}}+\log h-\int_{\Sigma} v \log h d v_{g}\right)
$$


as $k \rightarrow+\infty$. Sequently, we proceed in a normal coordinate system near $x_{k}$. Let $T\left(\psi_{k}, \phi_{k}\right)$ be a set of all smooth functions $u \in \mathbb{R}^{2}$ with $\left.u\right|_{\partial \mathbb{B}_{\delta}(0)}=\psi_{k}$ and $\left.u\right|_{\partial \mathbb{B}_{R r_{k}(0)}}=\phi_{k}$. It is not difficult to see that $\inf _{u \in T\left(\psi_{k}, \phi_{k}\right)} \int_{\mathbb{B}_{\delta}(0) \backslash \mathbb{B}_{R r_{k}}(0)}|\nabla u|^{2} d x$ is attained by some function $h$ satisfying $\Delta h=0$ in $\mathbb{B}_{\delta}(0) \backslash \mathbb{B}_{R r_{k}}(0)$ with $\left.h\right|_{\partial \mathbb{B}_{\delta}(0)}=\psi_{k},\left.h\right|_{\partial \mathbb{B}_{R r_{k}}(0)}=\phi_{k}$. Then it follows that

$$
h(x)=\frac{\phi_{k}(\log \delta-\log r)+\psi_{k}\left(\log r-\log R r_{k}\right)}{\log \delta-\log R r_{k}},
$$

and that

$$
\int_{\mathbb{B}_{\delta}(0) \backslash \mathbb{B}_{R r_{k}}(0)}|\nabla h|^{2} d x=\frac{2 \pi\left(\phi_{k}-\psi_{k}\right)^{2}}{\log \delta-\log R r_{k}}
$$

Define a function space

$$
\mathscr{W}_{k}\left(\psi_{k}, \phi_{k}\right)=\left\{v_{k} \in \mathscr{H}_{\mathbf{G}}^{2}\left(B_{\delta}\left(x_{k}\right) \backslash B_{R r_{k}}\left(x_{k}\right)\right):\left.v_{k}\right|_{\partial B_{\delta}\left(x_{k}\right)}=\psi_{k},\left.v_{k}\right|_{\partial B_{R r_{k}}\left(x_{k}\right)}=\phi_{k}\right\},
$$

where $\mathscr{H}_{\mathbf{G}}^{2}$ is in (1.8). Let $\widetilde{v}_{k}=\max \left\{\psi_{k}, \min \left\{v_{k}, \phi_{k}\right\}\right\}$. Then $\widetilde{v}_{k} \in \mathscr{W}_{k}\left(\psi_{k}, \phi_{k}\right)$ and in a normal coordinate system near $x_{k}$, there holds by

$$
\int_{B_{\delta}\left(x_{k}\right) \backslash B_{R r_{k}}\left(x_{k}\right)}\left|\nabla v_{k}\right|^{2} d v_{g} \geq \int_{B_{\delta}\left(x_{k}\right) \backslash B_{R r_{k}}\left(x_{k}\right)}\left|\nabla \widetilde{v}_{k}\right|^{2} d v_{g} \geq \int_{\mathbb{B}_{\delta}(0) \backslash \mathbb{B}_{R r_{k}}(0)}|\nabla h|^{2} d x .
$$

This together with $3.23,(3.24)$ and 3.26$)$, one can easily check that

$$
\begin{aligned}
& \int_{\bigcup_{i=1}^{\ell} B_{\delta}\left(\sigma_{i}\left(x_{k}\right)\right) \backslash B_{R r_{k}}\left(\sigma_{i}\left(x_{k}\right)\right)}\left|\nabla v_{k}\right|^{2} d v_{g} \\
\geq & \frac{2 \pi \ell\left(\phi_{k}-\psi_{k}\right)^{2}}{\log \delta-\log R r_{k}} \\
\geq & 2 \pi \ell\left(2+\frac{\bar{v}_{k}}{\log r_{k}}\right)^{2}\left(-\log r_{k}+\log R-\log \delta-\frac{C_{R, \delta}}{\log r_{k}}\right) \\
& +4 \pi \ell\left(2+\frac{\bar{v}_{k}}{\log r_{k}}\right)\left(\varphi_{k}+\frac{\varphi_{k} C_{R, \delta}}{\left(\log r_{k}\right)^{2}}\right)+\frac{\ell C_{R, \delta}^{\prime} \bar{v}_{k}}{2\left(\log r_{k}\right)^{2}}+o_{k}(1),
\end{aligned}
$$

where $C_{R, \delta}$ and $C_{R, \delta}^{\prime}$ are constants relying only on $\delta$ and $R$.

Finally, we compute the integral $\int_{\bigcup_{i=1}^{\ell} B_{R r_{k}}\left(\sigma_{i}\left(x_{k}\right)\right)}\left|\nabla v_{k}\right|^{2} d v_{g}$. Thanks to Proposition 3.1, we obtain

$$
\begin{aligned}
& \int_{\bigcup_{i=1}^{\ell} B_{R r_{k}}\left(\sigma_{i}\left(x_{k}\right)\right)}\left|\nabla v_{k}\right|^{2} d v_{g} \\
= & \ell\left(1+o_{k}(1)\right) \int_{B_{R}(0)}\left|\widetilde{v}_{\infty}(x)\right|^{2} d x \\
\geq & 16 \pi \ell\left(1+o_{k}(1)\right)\left(\log \left(1+\lambda^{2}\left(R-\left|\widetilde{x}_{0}\right|\right)^{2}\right)-\frac{\lambda^{2}\left(R-\left|\widetilde{x}_{0}\right|\right)^{2}}{1+\lambda^{2}\left(R-\left|\widetilde{x}_{0}\right|\right)^{2}}\right) .
\end{aligned}
$$


Inserting (3.18), 3.22), 3.27) and 3.28) into (3.16), we conclude that

$$
\begin{aligned}
J_{8 \pi \ell}\left(v_{k}\right) \geq & -\pi \ell \log r_{k}\left(2-\frac{\bar{v}_{k}}{\log r_{k}}\right)^{2}+\frac{\ell C_{R, \delta}^{\prime} \bar{v}_{k}}{2\left(\log r_{k}\right)^{2}}+2 \pi \ell\left(2+\frac{\bar{v}_{k}}{\log r_{k}}\right)\left(\varphi_{k}+\frac{C_{R, \delta} \varphi_{k}}{\left(\log r_{k}\right)^{2}}\right) \\
& -16 \pi \ell \log \delta+4 \pi \ell \widetilde{A}_{x_{0}}+\pi \ell\left(2+\frac{\bar{v}_{k}}{\log r_{k}}\right)^{2}\left(\log R-\log \delta-\frac{C_{R, \delta}}{\log r_{k}}\right) \\
& +8 \pi \ell\left(1+o_{k}(1)\right)\left(\log \left(1+\lambda^{2}\left(R-\left|\widetilde{x}_{0}\right|\right)^{2}\right)-\frac{\lambda^{2}\left(R-\left|\widetilde{x}_{0}\right|\right)^{2}}{1+\lambda^{2}\left(R-\left|\widetilde{x}_{0}\right|\right)^{2}}\right)+o_{\delta}(1) \\
& -\int_{\bigcup_{i=1}^{\ell} B_{\delta}\left(\sigma_{i}\left(x_{0}\right)\right)} \nabla \widetilde{G}_{x_{0}} \cdot \nabla \log h d v_{g} \\
& -\frac{1}{2} \int_{\Sigma \backslash \bigcup_{i=1}^{\ell} B_{\delta}\left(\sigma_{i}\left(x_{0}\right)\right)}|\nabla \log h|^{2} d v_{g}+o_{k}(1) .
\end{aligned}
$$

Using the divergence theorem, one has

$$
\begin{aligned}
& \int_{\bigcup_{i=1}^{\ell} B_{\delta}\left(\sigma_{i}\left(x_{0}\right)\right)} \nabla \widetilde{G}_{x_{0}} \cdot \nabla \log h d v_{g} \\
= & \ell\left(\int_{\partial B_{\delta}\left(x_{0}\right)} \frac{\partial \widetilde{G}_{x_{0}}}{\partial n} \log h d s_{g}-\int_{B_{\delta}\left(x_{0}\right)} \Delta \widetilde{G}_{x_{0}} \log h d v_{g}\right) \\
= & o_{\delta}(1) .
\end{aligned}
$$

Moreover, 3.29 implies that

$$
J_{8 \pi \ell}\left(v_{k}\right) \geq\left(C-\pi \ell \log r_{k}\right)\left(2-\frac{\bar{v}_{k}}{\log r_{k}}+O\left(-\frac{1}{\log r_{k}}\right)\right)^{2}+C .
$$

Note that $J_{8 \pi \ell}\left(v_{k}\right) \leq J_{8 \pi \ell}\left(v_{0}\right)$. Then it follows that

$$
\left|2-\frac{\bar{v}_{k}}{\log r_{k}}\right| \leq \frac{C}{\left(-\ell \log r_{k}\right)^{1 / 2}} .
$$

Letting $k \rightarrow+\infty$ leads to $\bar{v}_{k} / \log r_{k} \rightarrow 2$. Together with (3.25), 3.29) and (3.30), we finally arrive at

$$
\begin{aligned}
\lim _{k \rightarrow \infty} J_{8 \pi \ell}\left(v_{k}\right) \geq & -4 \pi \ell \max _{x \in \Sigma}\left(2 \log (\pi \ell h(x))+\widetilde{A}_{x}\right)-8 \pi \ell \\
& +8 \pi \ell \int_{\Sigma} \log h d v_{g}-\frac{1}{2} \int_{\Sigma}|\nabla \log h|^{2} d v_{g}
\end{aligned}
$$

by passing to the limit $k \rightarrow+\infty$ first and then $\delta \rightarrow 0, R \rightarrow+\infty$. Notice that $J_{8 \pi \ell}(v(t))$ decreases in $t$. According to 3.15 , we can find some $t_{0}>0$ such that

$$
\begin{aligned}
J_{8 \pi \ell}\left(v\left(t_{0}\right)\right)< & -4 \pi \ell \max _{x \in \Sigma}\left(2 \log (\pi \ell h(x))+\widetilde{A}_{x}\right)-8 \pi \ell \\
& +8 \pi \ell \int_{\Sigma} \log h d v_{g}-\frac{1}{2} \int_{\Sigma}|\nabla \log h|^{2} d v_{g}-\frac{\varepsilon}{2} .
\end{aligned}
$$


Then when $t_{k}>t_{0}$, we see that $J_{8 \pi \ell}\left(v\left(t_{k}\right)\right) \leq J_{8 \pi \ell}\left(v\left(t_{0}\right)\right)$, namely,

$$
\begin{aligned}
J_{8 \pi \ell}\left(v\left(t_{k}\right)\right)< & -4 \pi \ell \max _{x \in \Sigma}\left(2 \log (\pi \ell h(x))+\widetilde{A}_{x}\right)-8 \pi \ell \\
& +8 \pi \ell \int_{\Sigma} \log h d v_{g}-\frac{1}{2} \int_{\Sigma}|\nabla \log h|^{2} d v_{g},
\end{aligned}
$$

which contradicts with (3.31). Thus the proposition is proved.

\section{Completion of the proof of Theorem 1.1}

In this section, we will complete the proof of Theorem 1.1. Under the assumptions of Theorem 1.1. we shall construct a sequence of initial data $v_{0, \varepsilon}$ to show

$$
\begin{aligned}
J_{8 \pi \ell}\left(v_{0, \varepsilon}\right)< & -4 \pi \ell \max _{x \in \Sigma}\left(2 \log (\pi \ell h(x))+\widetilde{A}_{x}\right)-8 \pi \ell \\
& +8 \pi \ell \int_{\Sigma} \log h d v_{g}-\frac{1}{2} \int_{\Sigma}|\nabla \log h|^{2} d v_{g},
\end{aligned}
$$

where $\widetilde{A}_{x}$ is defined as in 3.11). Observe from 2.6 that $J_{8 \pi \ell}(v(t)) \leq J_{8 \pi \ell}\left(v_{0, \varepsilon}\right)$ as $t \rightarrow+\infty$. This yields to a contradiction with Proposition 3.5 . Therefore we conclude that $v_{k}$ is compact. Then we follow the idea of 3 to get the convergence of the flow. This finishes the proof of the theorem.

\subsection{Exclusion of blow-up phenomenon}

We first exclude the blow-up phenomenon. Pick up some point $p \in \Sigma$ such that

$$
2 \log (\pi \ell h(p))+\widetilde{A}_{p}=\max _{x \in \Sigma}\left(2 \log (\pi \ell h(x))+\widetilde{A}_{x}\right)
$$

Notice that $I(x)=\sharp \mathbf{G}(x) \equiv \ell$ for all $x \in \Sigma$ and $\mathbf{G}=\left\{\sigma_{1}, \ldots, \sigma_{\ell}\right\}$. Then $I(p)=\ell$ and $\sigma_{1}(p), \ldots, \sigma_{\ell}(p)$ are different points on $\Sigma$. For some $\delta>0$, choose a normal coordinate system $\left(B_{\delta}\left(x_{0}\right), \exp _{p}^{-1} ;\left\{y^{1}, y^{2}\right\}\right)$ near $p$. By $25, \widetilde{G}_{p}$ can be written as

$$
\widetilde{G}_{p}\left(\exp _{p}(y)\right)=-4 \log r+\widetilde{A}_{p}+b_{1} y^{1}+b_{2} y^{2}+c_{1}\left(y^{1}\right)^{2}+2 c_{2} y^{1} y^{2}+c_{3}\left(y^{2}\right)^{2}+O\left(r^{3}\right)
$$

where $r=|y|=d_{g}\left(p, \exp _{p}(y)\right), \widetilde{A}_{p}$ is a constant. Following the arguments of 25, Section 5], we define

$$
\phi_{\epsilon}(x)= \begin{cases}c-2 \log \left(1+\frac{r^{2}}{8 \epsilon^{2}}\right)+\widetilde{A}_{p}+\alpha\left(\exp _{p}^{-1}\left(\sigma_{i}^{-1}(x)\right)\right), & x \in B_{R \epsilon}\left(\sigma_{i}(p)\right), i=1, \ldots, \ell, \\ \widetilde{G}_{p}(x)-\eta\left(\sigma_{i}^{-1}(x)\right) \beta\left(\exp _{p}^{-1}\left(\sigma_{i}^{-1}(x)\right)\right), & x \in B_{2 R \epsilon}\left(\sigma_{i}(p)\right) \backslash B_{R \epsilon}\left(\sigma_{i}(p)\right), \\ \widetilde{G}_{p}(x), & x \in \Sigma \backslash \bigcup_{i=1}^{\ell} B_{2 R \epsilon}\left(\sigma_{i}(p)\right),\end{cases}
$$

where $\widetilde{A}_{p}$ is defined in 4.2$), R$ and $c$ are constants depending only on $\epsilon$ and will be determined later, $r=r(x)$ denotes the geodesic distance between $x$ and $\sigma_{i}(p)$ for $x \in$ 
$B_{R \epsilon}\left(\sigma_{i}(p)\right), \eta \in C_{0}^{\infty}\left(B_{2 R \epsilon}(p)\right)$ is a cut-off function, satisfying $0 \leq \eta \leq 1, \eta \equiv 1$ on $B_{R \epsilon}(p)$ and $\left|\nabla_{g} \eta\right| \leq 4 /(R \epsilon), \alpha(y)=b_{1} y^{1}+b_{2} y^{2}$ and $\beta(y)=c_{1}\left(y^{1}\right)^{2}+2 c_{2} y^{1} y^{2}+c_{3}\left(y^{2}\right)^{2}+O\left(r^{3}\right)$.

Set $\widetilde{v}_{0, \varepsilon}=\left(\phi_{\epsilon}-\bar{\phi}_{\epsilon}\right)+\log h$. In view of 1.6$)$, we obtain

$$
\begin{aligned}
J_{8 \pi \ell}\left(\widetilde{v}_{0, \varepsilon}\right)= & \frac{1}{2} \int_{\Sigma}\left|\nabla_{g} \phi_{\epsilon}\right|^{2} d v_{g}-8 \pi \ell \log \int_{\Sigma} h e^{\phi_{\epsilon}} d v_{g}+8 \pi \ell \bar{\phi}_{\epsilon} \\
& +8 \pi \ell \int_{\Sigma} \log h d v_{g}-\frac{1}{2} \int_{\Sigma}|\nabla \log h|^{2} d v_{g} .
\end{aligned}
$$

By the result of [25], it then follows from (4.3) that

$$
\begin{aligned}
J_{8 \pi \ell}\left(\widetilde{v}_{0, \varepsilon}\right)= & -8 \pi \ell-4 \pi \ell \widetilde{A}_{p}-8 \pi \ell \log (\pi \ell h(p)) \\
& -32 \pi \ell\left(8 \pi \ell-2 K(p)+b_{1}^{2}+b_{2}^{2}+\frac{\Delta h(p)}{h(p)}+\frac{2\left(k_{1} b_{1}+k_{2} b_{2}\right)}{h(p)}+o_{\epsilon}(1)\right) \epsilon^{2} \log \frac{1}{\epsilon} \\
& +8 \pi \ell \int_{\Sigma} \log h d v_{g}-\frac{1}{2} \int_{\Sigma}|\nabla \log h|^{2} d v_{g},
\end{aligned}
$$

where $b_{1}$ and $b_{2}$ are defined in 4.2$),\left(k_{1}, k_{2}\right)=\nabla h(p)$. Since $\Delta \log h=Q-8 \pi \ell$, there holds

$$
\frac{\Delta h(p)}{h(p)}=Q-8 \pi \ell+\frac{k_{1}^{2}+k_{2}^{2}}{h(p)^{2}} .
$$

Under the hypothesis $Q(p)>2 K(p)$, we have by 4.5 that

$$
\begin{aligned}
& 8 \pi \ell-2 K(p)+b_{1}^{2}+b_{2}^{2}+\frac{\Delta h(p)}{h(p)}+\frac{2\left(k_{1} b_{1}+k_{2} b_{2}\right)}{h(p)} \\
= & Q(p)-2 K(p)+\left(\frac{k_{1}+b_{1} h(p)}{h(p)}\right)^{2}+\left(\frac{k_{2}+b_{2} h(p)}{h(p)}\right)^{2}>0 .
\end{aligned}
$$

Inserting (4.6) into (4.4), by 4.1), we find

$$
\begin{aligned}
J_{8 \pi \ell}\left(\widetilde{v}_{0, \varepsilon}\right)< & -4 \pi \ell \max _{x \in \Sigma}\left(2 \log (\pi \ell h(x))+\widetilde{A}_{x}\right)-8 \pi \ell \\
& +8 \pi \ell \int_{\Sigma} \log h d v_{g}-\frac{1}{2} \int_{\Sigma}|\nabla \log h|^{2} d v_{g} .
\end{aligned}
$$

Observe that $\widetilde{v}_{0, \varepsilon}$ is the function of Lipschitz. Clearly, $\widetilde{v}_{0, \varepsilon}$ can be modified into a smooth function $\widehat{v}_{0, \varepsilon}$, and $J_{8 \pi \ell}\left(\widehat{v}_{0, \varepsilon}\right)$ satisfies (4.7). Then, choose some constant $c_{0}$ such that $\int_{\Sigma} e^{\widehat{v}_{0, \varepsilon}+c_{0}} d v_{g}=1$. Denote $v_{0, \varepsilon}=\widehat{v}_{0, \varepsilon}+c_{0}$. As a consequence,

$$
\begin{aligned}
J_{8 \pi \ell}\left(v_{0, \varepsilon}\right)< & -4 \pi \ell \max _{x \in \Sigma}\left(2 \log (\pi \ell h(x))+\widetilde{A}_{x}\right)-8 \pi \ell \\
& +8 \pi \ell \int_{\Sigma} \log h d v_{g}-\frac{1}{2} \int_{\Sigma}|\nabla \log h|^{2} d v_{g},
\end{aligned}
$$

which contradicts to 3.14. Thus, we conclude that blow-up can't happen and the sequence $v_{k}$ is compact. 


\subsection{The convergence}

We follow the ideas of Catéras in $[3$ for the study of convergence. As $k \rightarrow+\infty$, note that

$$
\begin{aligned}
\int_{\Sigma}\left(\Delta v_{k}-\Delta v_{\infty}\right)^{2} d v_{g} & =\int_{\Sigma}\left(8 \pi \ell\left(e^{v_{\infty}}-e^{v_{k}}\right)+\frac{\partial e^{v_{k}}}{\partial t}\right)^{2} d v_{g} \\
& \leq C \int_{\Sigma}\left(e^{v_{\infty}}-e^{v_{k}}\right)^{2} d v_{g}+C \int_{\Sigma}\left|\frac{\partial v_{k}}{\partial t}\right|^{2} e^{v_{k}} d v_{g} \rightarrow 0
\end{aligned}
$$

where $v_{\infty}$ is a solution of 1.10 . By the result of Simon [21, we finally obtain that

$$
\left\|v(t)-v_{\infty}\right\|_{H^{2}(\Sigma)} \rightarrow 0 \quad \text { as } k \rightarrow+\infty
$$

Therefore, Theorem 1.1 is established.

\section{References}

[1] T. Aubin, Nonlinear Analysis on Manifolds: Monge-Ampère equations, Grundlehren der Mathematischen Wissenschaften 252, Springer-Verlag, New York, 1982.

[2] J.-B. Castéras, A mean field type flow part I: Compactness of solutions to a perturbed mean field type equation, Calc. Var. Partial Differential Equations 53 (2015), no. 1-2, $221-246$.

[3] _ A mean field type flow II: Existence and convergence, Pacific J. Math. 276 (2015), no. 2, 321-345.

[4] S.-Y. A. Chang and P. C. Yang, Prescribing Gaussian curvature on $S^{2}$, Acta Math. 159 (1987), no. 3-4, 215-259.

[5] _ Conformal deformation of metrics on $S^{2}$, J. Differential Geom. 27 (1988), no. 2, 259-296.

[6] C.-C. Chen and C.-S. Lin, Sharp estimates for solutions of multi-bubbles in compact Riemann surfaces, Comm. Pure Appl. Math. 55 (2002), no. 6, 728-771.

[7] _ Topological degree for a mean field equation on Riemann surfaces, Comm. Pure Appl. Math. 56 (2003), no. 12, 1667-1727.

[8] W. X. Chen, A Trüdinger inequality on surfaces with conical singularities, Proc. Amer. Math. Soc. 108 (1990), no. 3, 821-832.

[9] W. Chen and W. Ding, Scalar curvatures on $S^{2}$, Trans. Amer. Math. Soc. 303 (1987), no. 1, 365-382. 
[10] A problem concerning the scalar curvature on $S^{2}$, Kexue Tongbao (Engl. Ed.) 33 (1988), 533-537.

[11] W. X. Chen and C. Li, Classification of solutions of some nonlinear elliptic equations, Duke Math. J. 63 (1991), no. 3, 615-622.

[12] W. Ding, J. Jost, J. Li and G. Wang, The differential equation $\Delta u=8 \pi-8 \pi h e^{u}$ on a compact Riemann surface, Asian J. Math. 1 (1997), no. 2, 230-248.

[13] _ Existence results for mean field equations, Ann. Inst. H. Poincaré Anal. Non Linéaire 16 (1999), no. 5, 653-666.

[14] Y. Fang and Y. Yang, Trudinger-Moser inequalities on a closed Riemannian surface with the action of a finite isometric group, Ann. Sc. Norm. Sup. Pisa Cl. Sci. 20 (2020), no. 4, 1295-1324.

[15] J. L. Kazdan and F. W. Warner, Curvature functions for compact 2-manifolds, Ann. of Math. (2) 99 (1974), 14-47.

[16] J. Li and C. Zhu, The convergence of the mean field type flow at a critical case, Calc. Var. Partial Differential Equations 58 (2019), no. 2, Paper No. 60, 18 pp.

[17] A. Malchiodi, Topological methods for an elliptic equation with exponential nonlinearities, Discrete Contin. Dyn. Syst. 21 (2008), no. 1, 277-294.

[18] J. Moser, A sharp form of an inequality by N. Trudinger, Indiana Univ. Math. J. 20 (1971), no. 11, 1077-1092.

[19] _ On a nonlinear problem in differential geometry, in: Dynamical Systems (Proc. Sympos., Univ. Bahia, Salvador, 1971), 273-280, Academic Press, New York, 1973.

[20] M. Nolasco and G. Tarantello, On a sharp Sobolev-type inequality on two-dimensional compact manifolds, Arch. Ration. Mech. Anal. 145 (1998), no. 2, 161-195.

[21] L. Simon, Asymptotics for a class of nonlinear evolution equations, with applications to geometric problems, Ann. of Math. (2) 118 (1983), no. 3, 525-571.

[22] M. Struwe and G. Tarantello, On multivortex solutions in Chern-Simons gauge theory, Boll. Unione Mat. Ital. Sez. B Artic. Ric. Mat. (8) 1 (1998), no. 1, 109-121.

[23] Y. Yang and X. Zhu, A remark on a result of Ding-Jost-Li-Wang, Proc. Amer. Math. Soc. 145 (2017), no. 9, 3953-3959. 
[24] Existence of solutions to a class of Kazdan-Warner equations on compact Riemannian surface, Sci. China Math. 61 (2018), no. 6, 1109-1128.

[25] _ Mean field equations on a closed Riemannian surface with the action of an isometric group, Internat. J. Math. 31 (2020), no. 9, 2050072, 26 pp.

Yamin Wang

Department of Mathematics, Renmin University of China, Beijing 100872, China E-mail address: 2017100918@ruc.edu.cn 